
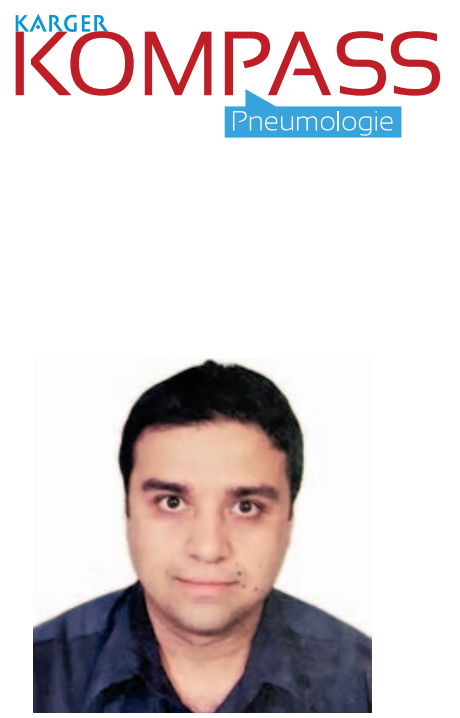

Dr. Fotios Drakopanagiotakis Medizinische Klinik und Poliklinik II Gießen

\title{
interstitiellen Lungenerkrankungen bei Patienten mit Sklerodermie
}

\author{
Ausgewählt und kommentiert von \\ Dr. Fotios Drakopanagiotakis (Gießen)
}

Interstitielle Lungenerkrankungen (ILD) gehören zu den Haupttypen der Lungenbeteiligung bei Patienten mit systemischer Sklerose (SSC). SSc-assoziierte ILD bestehen aus verschiedenen histopathologischen Subtypen, am häufigsten aus der nicht-spezifischen interstitiellen Pneumonie (non-specific interstitial pneumonia, NSIP) und seltener aus der gewöhnlichen interstitiellen Pneumonie (usual interstitial pneumonia, UIP). Patienten mit Lungenbeteiligung haben eine schlechtere Prognose als SSc-Patienten, die nicht betroffen sind.

Patienten mit diffusem Haut-SSc haben ein erhöhtes Risiko, ILD früh im Verlauf ihrer Krankheit zu entwickeln. Diese Gefahr besteht bei Patienten mit begrenztem kutanem SSc zwar weniger, jedoch kann in dem Fall ILD als eine späte Manifestation auftreten.

Die Lungenfunktion in der Diagnostik und Prognose der ILD bei Patienten mit SSC

Lungenfunktionstests werden bei der Mehrheit der Patienten mit SSC zum Zeitpunkt der Erstdiagnose durchgeführt, um festzustellen, ob SSc die Lunge beeinflusst hat und in welchem Ausmaß. Anschließend werden Lungenfunktionstests jährlich oder in kürzeren Abständen durchgeführt, um das Auftreten einer Dyspnoe, von Husten oder radiographischen Anomalien zu beobachten.

Patienten mit SSC und einer fast normalen FVC bei Erstvorstellung haben ein geringes Risiko für Progression zu schwerer ILD [1]. Ein normales Lungenvolumen jedoch kann das Frühstadium einer ILD nicht ausschließen [2].

Patienten, die keinen Rückgang der FVC über 4 Jahre haben, zeigen einen besseren ILD-Verlauf. Ein DLCO-Rückgang innerhalb eines Jahres von 15\% oder mehr ist ein schlechter prognostischer Faktor und identifiziert Patienten, die genauer beobachtet werden sollten [3].

Hochauflösende Computertomographie des Thorax

Die HRCT-Muster in den SSc-assoziierten ILD spiegeln die histopathologischen Muster der idiopathischen interstitiellen Pneumonien wider (z.B. fibrotische NSIP, UIP-Muster).

\section{KARGER}

Fax +497614520714

information@karger.com www.karger.com (c) 2017 S. Karger GmbH, Freiburg
Dr. Fotios Drakopanagiotakis

Medizinische Klinik und Poliklinik II

Klinikstraße 33, 35392 Gießen, Deutschland

Fotios.drakopanagiotakis@innere.med.uni-giessen.de 
Das Ausmaß der ILD, das sich in der hochauflösenden Computertomographie (HRCT) zeigt, ist mit der Mortalität und mit einer Verschlechterung der Lungenfunktion assoziiert [4-6].

Auch der Progress der fibrotischen Veränderungen im Krankheitsverlauf ist hoch prädiktiv für die Mortalität, wie eine prospektive Studie mit 215 Patienten gezeigt hat [7].

Noch effektiver in der Vorhersage der Mortalität ist die Kombination aus HRCT-Daten (ILD > 20\%) und Lungenfunktionsparametern (FVC <70\%). [7].

Eine Datenanalyse der Scleroderma Lung Study I [8] hat allerdings gezeigt, dass bei Patienten ohne pulmonale Hypertonie die DLCO und nicht die FVC der beste Parameter zur Einschätzung der Lungenbeteiligung gemäß HRCT ist.

\section{Therapie}

Die grundsätzliche Entscheidung über den Beginn einer Behandlung wird meist in Abhängigkeit von der Art und dem Ausmaß der pulmonalen Beteiligung getroffen. Bei einigen Patienten mit einer nur milden ILD wird daher lediglich der klinische Verlauf genau beobachtet und eine Therapie erst im Falle einer Verschlechterung funktioneller Parameter eingeleitet.

In den meisten Fällen werden die Glukokortikoide in einer niedrigen Dosierung (zur Vermeidung einer renalen Krise) mit anderen Immunsuppressiva kombiniert. Die Wirksamkeit von Kortikoiden zur Behandlung der SSc-assoziierten ILD ist unklar.
Cyclophosphamid hat eine gute Effektivität bei Sklerodermieassoziierten ILD gezeigt, wird aber aufgrund der potenziellen Nebenwirkungen (u.a. sekundäre Malignome, Zystitis, Knochenmarksdepression) meist erst bei fortgeschrittener ILD eingesetzt [9].

Mycophenolat mofetil (MMF) ist ein Inhibitor der Lymphozytenproliferation, der häufig bei der Behandlung von extrapulmonalen Manifestationen von SSc und anderen rheumatischen Erkrankungen verwendet wird. In der Scleroderma Lung Study II [10] wurde die Einnahme von MMF für 24 Monate mit oralem Cyclophosphamid für 12 Monate verglichen. Beide Gruppen zeigten eine Verbesserung der FVC, bei besserer Verträglichkeit von MMF gegenüber Cyclophosphamid.

Trotz des Einsatzes dieser Immunsuppressiva kann bei einigen Patienten ein weiterer Krankheitsprogress nicht verhindert werden. Nach Ausschluss von alternativen Krankheitsprozessen, kommen alternative Therapien in Frage. Hierzu zählt die Gabe von Rituximab [11]. Auch der Einsatz von Pirfenidon, das im Rahmen der idiopathischen pulmonalen Fibrose Wirksamkeit gezeigt hat, wird in Studien untersucht [12].

Zusammenfassend ist festzustellen, dass die Kombination aus lungenfunktionellen Parametern und HRCT-Auswertungen zum Ausmaß der Lungenbeteiligung prädiktiv für die Mortalität ist. Eine Erweiterung der therapeutischen Optionen gibt Hoffnung für die Behandlung von Patienten mit SSc-assoziierter ILD.

\section{Literatur}

1 Plastiras SC, Karadimitrakis SP, Ziakas PD, et al.: Scleroderma lung: initial forced vital capacity as predictor of pulmonary function decline. Arthritis Rheum 2006;55:598.

2 Suliman YA, Dobrota R, Huscher D, et al.: Brief report: pulmonary function tests: high rate of false-negative results in the early detection and screening of scleroderma-related interstitial lung disease. Arthritis Rheumatol 2015;67:3256-3261.

3 Moore OA, Proudman SM, Goh N, et al.: Quantifying change in pulmonary function as a prognostic marker in systemic sclerosis-related interstitial lung disease. Clin Exp Rheumatol 2015;33(4 suppl 91):S111-S116.

4 Moore OA, Goh N, Corte T, et al.: Extent of disease on high-resolution computed tomography lung is a predictor of decline and mortality in systemic sclerosis-related interstitial lung disease. Rheumatology (Oxford) 2013;52: 155-160.
5 Hoffmann-Vold AM, Aalokken TM, Lund MB, et al.: Predictive value of serial high-resolution computed tomography analyses and concurrent lung function tests in systemic sclerosis. Arthritis Rheumatol 2015;67:2205-2212.

6 Khanna D, Nagaraja V, Tseng $\mathrm{CH}$, et al.: Predictors of lung function decline in scleroderma-related interstitial lung disease based on high-resolution computed tomography: implications for cohort enrichment in systemic sclerosis-associated interstitial lung disease trials. Arthritis Res Ther 2015;23:372.

7 Goh NS, Desai SR, Veeraraghavan S, et al.: Interstitial lung disease in systemic sclerosis: a simple staging system. Am J Respir Crit Care Med 2008;177:1248.

8 Tashkin DP, Volkmann ER, Tseng $\mathrm{CH}$, et al.: Relationship between quantitative radiographic assessments of interstitial lung disease and physiological and clinical features of systemic sclerosis. Ann Rheum Dis 2016;75:374-381.
9 Tashkin DP, Elashoff R, Clements PJ, et al.: Cyclophosphamide versus placebo in scleroderma lung disease. N Engl J Med 2006;354: 2655-2666.

10 Tashkin DP, Roth MD, Clements PJ, et al.: Mycophenolate mofetil versus oral cyclophosphamide in scleroderma-related interstitial lung disease (SLS II): a randomised controlled, double-blind, parallel group trial. Lancet Respir Med 2016;4:708-719.

11 Jordan S, Distler JH, Maurer B, et al.: Effects and safety of rituximab in systemic sclerosis: an analysis from the European Scleroderma Trial and Research (EUSTAR) group. Ann Rheum Dis 2015;74:1188-1194.

12 Khanna D, Albera C, Fischer A, et al.: An open-label, phase II study of the safety and tolerability of pirfenidone in patients with scleroderma-associated interstitial lung disease: the LOTUSS Trial. J Rheumatol 2016;43: 1672-1679. 